\title{
A CAPITULATION PROBLEM AND GREENBERG'S CONJECTURE ON REAL QUADRATIC FIELDS
}

\author{
T. FUKUDA AND K. KOMATSU
}

\begin{abstract}
We give a sufficient condition in order that an ideal of a real quadratic field $F$ capitulates in the cyclotomic $\mathbb{Z}_{3}$-extension of $F$ by using a unit of an intermediate field. Moreover, we give new examples of $F$ 's for which Greenberg's conjecture holds by calculating units of fields of degree $6,18,54$ and 162 .
\end{abstract}

\section{INTRODUCTION}

Let $p$ be a prime number, $F$ a totally real number field, $F_{\infty}$ the cyclotomic $\mathbb{Z}_{p^{-}}$ extension of $F$ and $F_{n}$ the $n$th layer of $F_{\infty} / F$. Let $A_{n}$ be the $p$-part of the ideal class group of $F_{n}$. In [1], Greenberg showed the following:

Proposition . Assume that only one prime of $F$ lies over $p$ and that this prime is totally ramified in $F_{\infty} / F$. Then the following two statements are equivalent.

(1) Every ideal class of $A_{0}$ becomes trivial in $A_{n}$ for some $n$.

(2) The order of $A_{n}$ is bounded as $n \rightarrow \infty$.

In this paper, we treat the case that $F$ is a real quadratic field and $p=3$. In $\S 2$ we give a sufficient condition for (1) by using a unit in $F_{n}$. In $\S 3$ we give a method of finding the above unit.

\section{THEOREM}

We put $\zeta_{3^{n}}=e^{2 \pi \sqrt{-1} / 3^{n}}$ for a positive integer $n$. Our main purpose of this section is to prove the following theorem which plays a fundamental role in the next section.

Theorem . Let $F$ be a real quadratic field. Let $F_{n}=F\left(\zeta_{3^{n+1}}\right) \cap \mathbb{R}, G\left(F_{n} / \mathbb{Q}\right)=$ $\langle\sigma\rangle$ the Galois group $F_{n}$ over $\mathbb{Q}, \varepsilon$ a fundamental unit of $F$ and $A_{n}$ the 3-part of the ideal class group of $F_{n}$. We assume that 3 divides the class number $h_{F}$ of $F$ and that 3 does not split in $F / \mathbb{Q}$. If there exists a unit $\eta$ of $F_{n}$ such that $\eta^{1+\sigma}$ is a cube of an element of $F_{n}$ and that neither $\eta$ nor $\eta \varepsilon$ nor $\eta \varepsilon^{2}$ is a cube of an element of $F_{n}$, then the natural mapping of $A_{0}$ to $A_{n}$ is not injective.

Let $F_{n}^{*}=F\left(\zeta_{3^{n+1}}\right)$ and $F^{\prime}$ be the imaginary quadratic field contained in $F_{0}^{*}$ such that $F^{\prime} \cap \mathbb{Q}(\sqrt{-3})=\mathbb{Q}$. Let $M$ be the maximal abelian 3-extension of $F_{0}^{*}$ unramified outside $3, X=G\left(M / F^{\prime}\right)$ and $\rho$ the complex conjugation. We put

Received by the editor September 26, 1994 and, in revised form, February 11, 1995.

1991 Mathematics Subject Classification. Primary 11R30, 11R22, 11Y40.

Key words and phrases. Iwasawa invariants, real quadratic fields, unit groups, computation.

(C)1996 American Mathematical Society 
$X^{+}=\left\{x \in X \mid \rho^{-1} x \rho=x\right\}$. Let $M^{-}$be the intermediate field between $F_{0}^{*}$ and $M$ corresponding to $X^{+}$. For a real number $\alpha$, we denote by $\sqrt[3]{\alpha}$ the real number whose cube is $\alpha$. Even though the following Lemma 2.1 is well known, for completeness we give a proof.

Lemma 2.1. Let $\alpha$ be an element of $F$. If $F_{0}^{*}(\sqrt[3]{\alpha}) \subset M$, then $F_{0}^{*}(\sqrt[3]{\alpha}) \subset M^{-}$.

Proof. Let $\sigma$ be an element of $X^{+}$with $\sqrt[3]{\alpha}=\sqrt[3]{\alpha} \zeta$, where $\zeta$ is a cubic root of unity. Then we have $\sqrt[3]{\alpha}{ }^{\rho \sigma \rho^{-1}}=(\sqrt[3]{\alpha} \zeta)^{\rho^{-1}}=\sqrt[3]{\alpha} \zeta^{-1}=\sqrt[3]{\alpha}=\sqrt[3]{\alpha} \zeta$. Hence we have $\zeta=1$. This shows $\sqrt[3]{\alpha} \in M^{-}$.

For an ideal $\mathfrak{A}$ of $F$, we denote by $\overline{\mathfrak{A}}$ the ideal class of $F$ which contains $\mathfrak{A}$. Let $\overline{\mathfrak{A}}_{1}, \ldots, \overline{\mathfrak{A}}_{r}$ be a basis of $\left\{a \in A_{0} \mid a^{3}=1\right\}, \mathfrak{A}_{i}^{3}=\left(\alpha_{i}\right)$ and $k$ the intermediate field between $F_{0}^{*}$ and $M$ corresponding to $X^{3}=\left\{x^{3} \mid x \in X\right\}$. Then under the assumption that 3 does not split in $F / \mathbb{Q}$ we have by Lemma 2.1 the following result.

Lemma 2.2 (cf. [1, p. 281]). Let $k^{-}$be the field $k \cap M^{-}$. Then we have $k^{-}=$ $F_{0}^{*}\left(\sqrt[3]{3}, \sqrt[3]{\varepsilon}, \sqrt[3]{\alpha_{1}}, \ldots, \sqrt[3]{\alpha_{r}}\right)$.

The following is well known (cf. [1, p. 280]):

Lemma 2.3. Let $\sigma$ be a generator of the Galois group $G\left(F_{n}^{*} / F^{\prime}\right)$ and $\alpha$ be a nonzero element of $F_{n}^{*}$ such that there exists an element $\beta$ with $\alpha^{\sigma}=\alpha^{-1} \beta^{3}$. Then $F_{n}^{*}(\sqrt[3]{\alpha})$ is an abelian extension of $F^{\prime}$.

Proof of the Theorem. Since $\eta^{1-\sigma^{2}}=\left(\eta^{1+\sigma}\right)^{1-\sigma}$, there exists an element $\beta$ of $F_{n}$ with $\eta^{1-\sigma^{2}}=\beta^{3}$. Hence we have $N_{F_{n} / F_{0}}\left(\beta^{3}\right)=1$, which means $N_{F_{n} / F_{0}}(\beta)=1$. Hence there exists an element $\gamma$ of $F_{n}$ with $\beta=\gamma^{1-\sigma^{2}}$, which shows $\eta \gamma^{-3} \in F_{0}$. This shows $F_{n}^{*}(\sqrt[3]{\eta})=F_{n}^{*}\left(\sqrt[3]{\eta \gamma^{-3}}\right)=F_{n}^{*} F_{0}^{*}\left(\sqrt[3]{\eta \gamma^{-3}}\right)$. Since $F_{n}^{*}(\sqrt[3]{\eta})$ is an abelian 3-extension of $F_{0}^{*}$ unramified outside 3 by Lemma 2.3 and since $\eta \gamma^{-3} \in F_{0}^{*}$, we have $F_{0}^{*}\left(\sqrt[3]{\eta \gamma^{-3}}\right) \subset k^{-}=F_{0}^{*}\left(\sqrt[3]{3}, \sqrt[3]{\varepsilon}, \sqrt[3]{\alpha_{1}}, \ldots, \sqrt[3]{\alpha_{r}}\right)$ by Lemmas 2.1 and 2.2 . Hence there exist integers $n_{1}, n_{2}, \ldots, n_{r}, n$ and an element $\delta$ of $F_{0}$ with $\eta \gamma^{-3}=$ $\alpha_{1}^{n_{1}} \cdots \alpha_{r}^{n_{r}} \varepsilon^{n} \delta^{3}$ by Lemma 2.2. This shows by the assumption on $\eta$ that $\mathfrak{A}_{1}^{n_{1}} \cdots \mathfrak{A}_{r}^{n_{r}}$ is not principal in $F_{0}$ but principal in $F_{n}$.

\section{Method of Finding $\eta$}

In this section, we explain how to compute and find a unit $\eta$ in the theorem. Let $E_{n}$ be the unit group of $F_{n}$ and $r=2 \cdot 3^{n}-1$. If a basis $\left\{\varepsilon_{1} E_{n}^{3}, \ldots, \varepsilon_{r} E_{n}^{3}\right\}$ of $E_{n} / E_{n}^{3}$ is obtained, without loss of generality, $\eta$ can be written in the form $\eta=\varepsilon_{1}^{e_{1}} \cdots \varepsilon_{r}^{e_{r}}$ with $0 \leq e_{i} \leq 2$. Therefore, we can decide whether or not such an $\eta$ exists by examining all the combinations of $\left\{e_{1}, \ldots, e_{r}\right\}$. If $n=1$, we can obtain fundamental units of $F_{1}$ (cf. [3]) and can use this direct algorithm. But it is hard to obtain a basis of $E_{n} / E_{n}^{3}$ for $n \geq 2$. So we proceed as follows.

For an element $\xi$ of $F_{n}$, we denote $\xi^{\sigma^{i}}$ by $\xi_{i}$. Let $C_{n}$ be the cyclotomic unit group of $F_{n}$. First we assume that there exists an element $\xi \in C_{n}$ such that $C_{n}=$ $\left\langle-1, \xi_{0}, \ldots, \xi_{r-1}\right\rangle$. Moreover, we assume that the 3-Sylow subgroup $\left(E_{n} / C_{n}\right)_{3}$ of $E_{n} / C_{n}$ is cyclic of order $3^{n}$. Under these assumptions, we determine the form of $\alpha \in E_{n}$ which satisfies $\left(E_{n} / C_{n}\right)_{3}=\left\langle\alpha C_{n}\right\rangle$ and $\alpha^{1+\sigma} \in E_{n}^{3}$. From the assumption $A_{0} \neq 1$, there exists $\gamma \in E_{0}$ such that

$$
\gamma^{3}=\prod_{i=0}^{3^{n}-1} \xi_{2 i} .
$$


Assume that $\left(E_{n} / C_{n}\right)_{3}=\left\langle\alpha C_{n}\right\rangle$ and $\alpha^{1+\sigma}=\beta^{3}$ for some $\beta \in E_{n}$. Since the order of $\left(E_{n} / C_{n}\right)_{3}$ is $3^{n}$, we see that $\alpha^{3^{n-1}}=\gamma u, \beta=\alpha^{e} v$ for some $u, v \in C_{n}$ and $e \in \mathbb{N}$. Then

$$
u^{1+\sigma}= \pm\left(\alpha^{3^{n-1}}\right)^{1+\sigma}= \pm \beta^{3^{n}}= \pm \alpha^{e 3^{n}} v^{3^{n}} \equiv(\gamma u)^{3 e}=\prod_{i=0}^{3^{n}-1} \xi_{2 i}^{e} u^{3 e} \quad\left(\bmod C_{n}^{3^{n}}\right) .
$$

We write $u=\xi_{0}^{e_{0}} \cdots \xi_{r-1}^{e_{r-1}}$ with $e_{i} \in \mathbb{Z}$ and substitute this in both sides of the above congruence relation. Since $\xi_{r}= \pm\left(\xi_{0} \cdots \xi_{r-1}\right)^{-1}$, we obtain the following system of simultaneous equations:

$$
e_{i-1}+e_{i}-e_{r-1} \equiv \begin{cases}e+3 e e_{i} & \text { if } i \text { is even } \\ 3 e e_{i} & \text { if } i \text { is odd }\end{cases}
$$

Here the congruence is modulo $3^{n}$ and $e_{-1}=0$. This equation is easily solved. In fact, if we put $x=e_{r-1}$ and $y=e$, then we can represent all $e_{i}$ by $x$ and $y$. Now, we fix $x$ to be 0 and vary $y$ from 0 to $3^{n}-1$. If we find that $\gamma u$ is contained in $E_{n}^{3^{n-1}}$ for some $y$, then we put $\eta=(\gamma u)^{1 / 3^{n-1}}$. It is easy to check whether $\eta, \eta \varepsilon$ or $\eta \varepsilon^{2}$ is a cube in $E_{n}$.

A Galois generator $\xi$ of $C_{n}$ is hard to find. But we know the cyclotomic unit of Hasse (cf. [2]) which generates a fairly large subgroup of $C_{n}$. So, we execute the above procedure by letting $\xi$ to be Hasse's unit. We will be able to find $\eta$ by this method with some luck.

\section{EXAMPLES}

Let $F=\mathbb{Q}(\sqrt{m})$ where $m$ is a positive square-free integer congruent to 2 modulo 3. There are $207 \mathrm{~m}$ 's less than 10000 which satisfy $\left|A_{0}\right|=3$. We denote $\operatorname{Ker}\left(A_{0} \longrightarrow A_{n}\right)$ by $H_{n}$. We used a computer to implement the above method for these $F$ 's and fortunately found $\eta$ and conclude that $H_{n} \neq 1$ for many $F$ 's. We show the results of our computation in Table 1 (next page). The proposition in $\S 1$ implies that if $m \equiv 2(\bmod 3),\left|A_{0}\right|=3$, and $H_{n} \neq 1$ for some $n \geq 1$, then the order of $A_{n}$ is bounded, namely, Greenberg's conjecture is valid for $F$, and the Iwasawa invariant $\lambda_{3}(F)$ is zero. A question mark in the table means that we do not know the value. For example, we got $\left|H_{1}\right|=1$ when $m=899$ (cf. the remark below). So we searched $\eta \in F_{2}$ with the method of $\S 3$ but could not find it. We cannot conclude whether $\left|H_{2}\right|$ is 1 or 3 . Next we pursued a calculation in $F_{3}$ and found $\eta \in F_{3}$. Therefore $\left|H_{3}\right|=3$ and $\lambda_{3}(F)=0$.

Remark. Since $\left|H_{1}\right|=\left(E_{0}: N_{F_{1} / F_{0}}\left(E_{1}\right)\right)$, we can obtain the exact value of $\left|H_{1}\right|$ by computing $E_{1}$ (cf. [3]). We note that $\left|H_{1}\right|=1$ for all $m$ 's in Table 1 for which we could not find $\eta \in E_{1}$. 
TABLE 1 . All $m$ 's satisfying $m \equiv 2(\bmod 3)$ and $\left|A_{0}\right|=3(m<10000)$

\begin{tabular}{|c|c|c|c|c|c|c|c|c|c|c|c|}
\hline$m$ & $\left|H_{1}\right|$ & $\left|H_{2}\right|$ & $\left|H_{3}\right|$ & $\left|H_{4}\right|$ & $\lambda_{3}(F)$ & $m$ & $\left|H_{1}\right|$ & $\left|H_{2}\right|$ & $\left|H_{3}\right|$ & $\left|H_{4}\right|$ & $\lambda_{3}(F)$ \\
\hline 254 & 1 & $?$ & $?$ & $?$ & $?$ & 3221 & 3 & 3 & 3 & 3 & 0 \\
\hline 257 & 3 & 3 & 3 & 3 & 0 & 3281 & 3 & 3 & 3 & 3 & 0 \\
\hline 326 & 3 & 3 & 3 & 3 & 0 & 3287 & 3 & 3 & 3 & 3 & 0 \\
\hline 359 & 3 & 3 & 3 & 3 & 0 & 3305 & 1 & $?$ & $?$ & ? & $?$ \\
\hline 443 & 1 & 3 & 3 & 3 & 0 & 3419 & 3 & 3 & 3 & 3 & 0 \\
\hline 473 & 1 & $?$ & $?$ & $?$ & $?$ & 3422 & 1 & 3 & 3 & 3 & 0 \\
\hline 506 & 3 & 3 & 3 & 3 & 0 & 3482 & 3 & 3 & 3 & 3 & 0 \\
\hline 659 & 3 & 3 & 3 & 3 & 0 & 3569 & 1 & $?$ & 3 & 3 & 0 \\
\hline 761 & 3 & 3 & 3 & 3 & 0 & 3590 & 3 & 3 & 3 & 3 & 0 \\
\hline 785 & 1 & $?$ & 3 & 3 & 0 & 3602 & 3 & 3 & 3 & 3 & 0 \\
\hline 839 & 3 & 3 & 3 & 3 & 0 & 3803 & 3 & 3 & 3 & 3 & 0 \\
\hline 842 & 3 & 3 & 3 & 3 & 0 & 3941 & 3 & 3 & 3 & 3 & 0 \\
\hline 899 & 1 & $?$ & 3 & 3 & 0 & 3962 & 3 & 3 & 3 & 3 & 0 \\
\hline 1091 & 3 & 3 & 3 & 3 & 0 & 4001 & 3 & 3 & 3 & 3 & 0 \\
\hline 1211 & 3 & 3 & 3 & 3 & 0 & 4094 & 3 & 3 & 3 & 3 & 0 \\
\hline 1223 & 3 & 3 & 3 & 3 & 0 & 4106 & 3 & 3 & 3 & 3 & 0 \\
\hline 1229 & 3 & 3 & 3 & 3 & 0 & 4151 & 3 & 3 & 3 & 3 & 0 \\
\hline 1367 & 3 & 3 & 3 & 3 & 0 & 4193 & 3 & 3 & 3 & 3 & 0 \\
\hline 1373 & 3 & 3 & 3 & 3 & 0 & 4238 & 1 & 3 & 3 & 3 & 0 \\
\hline 1406 & 3 & 3 & 3 & 3 & 0 & 4283 & 3 & 3 & 3 & 3 & 0 \\
\hline 1478 & 3 & 3 & 3 & 3 & 0 & 4286 & 1 & $?$ & 3 & 3 & 0 \\
\hline 1523 & 3 & 3 & 3 & 3 & 0 & 4355 & 3 & 3 & 3 & 3 & 0 \\
\hline 1646 & 1 & $?$ & $?$ & $?$ & $?$ & 4367 & 3 & 3 & 3 & 3 & 0 \\
\hline 1787 & 3 & 3 & 3 & 3 & 0 & 4481 & 1 & 3 & 3 & 3 & 0 \\
\hline 1811 & 1 & 3 & 3 & 3 & 0 & 4493 & 3 & 3 & 3 & 3 & 0 \\
\hline 1847 & 3 & 3 & 3 & 3 & 0 & 4511 & 1 & 3 & 3 & 3 & 0 \\
\hline 1901 & 3 & 3 & 3 & 3 & 0 & 4649 & 3 & 3 & 3 & 3 & 0 \\
\hline 1907 & 3 & 3 & 3 & 3 & 0 & 4670 & 3 & 3 & 3 & 3 & 0 \\
\hline 1937 & 1 & $?$ & $?$ & $?$ & $?$ & 4706 & 3 & 3 & 3 & 3 & 0 \\
\hline 2021 & 1 & $?$ & 3 & 3 & 0 & 4778 & 3 & 3 & 3 & 3 & 0 \\
\hline 2099 & 1 & 3 & 3 & 3 & 0 & 4841 & 3 & 3 & 3 & 3 & 0 \\
\hline 2177 & 3 & 3 & 3 & 3 & 0 & 4853 & 3 & 3 & 3 & 3 & 0 \\
\hline 2207 & 3 & 3 & 3 & 3 & 0 & 4886 & 3 & 3 & 3 & 3 & 0 \\
\hline 2213 & 3 & 3 & 3 & 3 & 0 & 4907 & 1 & 3 & 3 & 3 & 0 \\
\hline 2429 & 1 & $?$ & 3 & 3 & 0 & 4910 & 3 & 3 & 3 & 3 & 0 \\
\hline 2459 & 3 & 3 & 3 & 3 & 0 & 4934 & 3 & 3 & 3 & 3 & 0 \\
\hline 2495 & 3 & 3 & 3 & 3 & 0 & 4970 & 3 & 3 & 3 & 3 & 0 \\
\hline 2510 & 1 & $?$ & 3 & 3 & 0 & 4982 & 3 & 3 & 3 & 3 & 0 \\
\hline 2543 & 3 & 3 & 3 & 3 & 0 & 4994 & 3 & 3 & 3 & 3 & 0 \\
\hline 2666 & 1 & $?$ & $?$ & 3 & 0 & 5042 & 3 & 3 & 3 & 3 & 0 \\
\hline 2678 & 1 & 3 & 3 & 3 & 0 & 5063 & 1 & $?$ & $?$ & $?$ & $?$ \\
\hline 2711 & 3 & 3 & 3 & 3 & 0 & 5081 & 1 & ? & ? & 3 & 0 \\
\hline 2726 & 3 & 3 & 3 & 3 & 0 & 5099 & 3 & 3 & 3 & 3 & 0 \\
\hline 2777 & 1 & 3 & 3 & 3 & 0 & 5102 & 3 & 3 & 3 & 3 & 0 \\
\hline 2831 & 3 & 3 & 3 & 3 & 0 & 5255 & 3 & 3 & 3 & 3 & 0 \\
\hline 2894 & 3 & 3 & 3 & 3 & 0 & 5261 & 3 & 3 & 3 & 3 & 0 \\
\hline 2918 & 1 & $?$ & 3 & 3 & 0 & 5297 & 1 & $?$ & $?$ & 3 & 0 \\
\hline 2981 & 3 & 3 & 3 & 3 & 0 & 5303 & 3 & 3 & 3 & 3 & 0 \\
\hline 2993 & 3 & 3 & 3 & 3 & 0 & 5327 & 3 & 3 & 3 & 3 & 0 \\
\hline 3023 & 3 & 3 & 3 & 3 & 0 & 5333 & 3 & 3 & 3 & 3 & 0 \\
\hline 3035 & 3 & 3 & 3 & 3 & 0 & 5369 & 3 & 3 & 3 & 3 & 0 \\
\hline 3047 & 1 & $?$ & $?$ & 3 & 0 & 5477 & 3 & 3 & 3 & 3 & 0 \\
\hline 3062 & 3 & 3 & 3 & 3 & 0 & 5621 & 3 & 3 & 3 & 3 & 0 \\
\hline 3071 & 3 & 3 & 3 & 3 & 0 & 5738 & 3 & 3 & 3 & 3 & 0 \\
\hline 3158 & 1 & $?$ & 3 & 3 & 0 & 5741 & 3 & 3 & 3 & 3 & 0 \\
\hline 3173 & 3 & 3 & 3 & 3 & 0 & 5798 & 3 & 3 & 3 & 3 & 0 \\
\hline
\end{tabular}


TABLE 1 (continued)

\begin{tabular}{|c|c|c|c|c|c|c|c|c|c|c|c|}
\hline$m$ & $\left|H_{1}\right|$ & $\left|H_{2}\right|$ & $\left|H_{3}\right|$ & $\left|H_{4}\right|$ & $\lambda_{3}(F)$ & $m$ & $\left|H_{1}\right|$ & $\left|H_{2}\right|$ & $\left|H_{3}\right|$ & $\left|H_{4}\right|$ & $\lambda_{3}(F)$ \\
\hline 5903 & 3 & 3 & 3 & 3 & 0 & 8282 & 1 & $?$ & 3 & 3 & 0 \\
\hline 5918 & 3 & 3 & 3 & 3 & 0 & 8285 & 3 & 3 & 3 & 3 & 0 \\
\hline 5930 & 3 & 3 & 3 & 3 & 0 & 8306 & 3 & 3 & 3 & 3 & 0 \\
\hline 5954 & 1 & ? & 3 & 3 & 0 & 8339 & 1 & ? & $?$ & 3 & 0 \\
\hline 6026 & 3 & 3 & 3 & 3 & 0 & 8363 & 1 & 3 & 3 & 3 & 0 \\
\hline 6053 & 3 & 3 & 3 & 3 & 0 & 8399 & 3 & 3 & 3 & 3 & 0 \\
\hline 6185 & 3 & 3 & 3 & 3 & 0 & 8426 & 3 & 3 & 3 & 3 & 0 \\
\hline 6209 & 3 & 3 & 3 & 3 & 0 & 8438 & 3 & 3 & 3 & 3 & 0 \\
\hline 6311 & 3 & 3 & 3 & 3 & 0 & 8447 & 3 & 3 & 3 & 3 & 0 \\
\hline 6401 & 3 & 3 & 3 & 3 & 0 & 8519 & 3 & 3 & 3 & 3 & 0 \\
\hline 6515 & 3 & 3 & 3 & 3 & 0 & 8543 & 3 & 3 & 3 & 3 & 0 \\
\hline 6557 & 3 & 3 & 3 & 3 & 0 & 8597 & 3 & 3 & 3 & 3 & 0 \\
\hline 6623 & 3 & 3 & 3 & 3 & 0 & 8603 & 3 & 3 & 3 & 3 & 0 \\
\hline 6686 & 3 & 3 & 3 & 3 & 0 & 8711 & 1 & $?$ & $?$ & ? & $?$ \\
\hline 6770 & 3 & 3 & 3 & 3 & 0 & 8735 & 3 & 3 & 3 & 3 & 0 \\
\hline 6782 & 3 & 3 & 3 & 3 & 0 & 8789 & 3 & 3 & 3 & 3 & 0 \\
\hline 6791 & 1 & 3 & 3 & 3 & 0 & 8837 & 1 & 3 & 3 & 3 & 0 \\
\hline 6806 & 1 & $?$ & $?$ & $?$ & $?$ & 8909 & 3 & 3 & 3 & 3 & 0 \\
\hline 6887 & 3 & 3 & 3 & 3 & 0 & 8930 & 3 & 3 & 3 & 3 & 0 \\
\hline 6995 & 1 & $?$ & ? & ? & $?$ & 8999 & 3 & 3 & 3 & 3 & 0 \\
\hline 7019 & 3 & 3 & 3 & 3 & 0 & 9062 & 3 & 3 & 3 & 3 & 0 \\
\hline 7055 & 3 & 3 & 3 & 3 & 0 & 9086 & 3 & 3 & 3 & 3 & 0 \\
\hline 7058 & 3 & 3 & 3 & 3 & 0 & 9149 & 3 & 3 & 3 & 3 & 0 \\
\hline 7235 & 3 & 3 & 3 & 3 & 0 & 9155 & 3 & 3 & 3 & 3 & 0 \\
\hline 7259 & 3 & 3 & 3 & 3 & 0 & 9215 & 3 & 3 & 3 & 3 & 0 \\
\hline 7262 & 3 & 3 & 3 & 3 & 0 & 9218 & 3 & 3 & 3 & 3 & 0 \\
\hline 7310 & 3 & 3 & 3 & 3 & 0 & 9278 & 3 & 3 & 3 & 3 & 0 \\
\hline 7319 & 3 & 3 & 3 & 3 & 0 & 9281 & 3 & 3 & 3 & 3 & 0 \\
\hline 7415 & 3 & 3 & 3 & 3 & 0 & 9293 & 3 & 3 & 3 & 3 & 0 \\
\hline 7481 & 3 & 3 & 3 & 3 & 0 & 9323 & 3 & 3 & 3 & 3 & 0 \\
\hline 7598 & 1 & $?$ & 3 & 3 & 0 & 9413 & 3 & 3 & 3 & 3 & 0 \\
\hline 7601 & 1 & $?$ & 3 & 3 & 0 & 9419 & 3 & 3 & 3 & 3 & 0 \\
\hline 7643 & 1 & 3 & 3 & 3 & 0 & 9467 & 3 & 3 & 3 & 3 & 0 \\
\hline 7655 & 3 & 3 & 3 & 3 & 0 & 9479 & 3 & 3 & 3 & 3 & 0 \\
\hline 7658 & 1 & $?$ & $?$ & 3 & 0 & 9551 & 3 & 3 & 3 & 3 & 0 \\
\hline 7673 & 3 & 3 & 3 & 3 & 0 & 9578 & 1 & 3 & 3 & 3 & 0 \\
\hline 7694 & 3 & 3 & 3 & 3 & 0 & 9590 & 1 & ? & ? & 3 & 0 \\
\hline 7709 & 1 & 3 & 3 & 3 & 0 & 9659 & 1 & 3 & 3 & 3 & 0 \\
\hline 7721 & 3 & 3 & 3 & 3 & 0 & 9710 & 3 & 3 & 3 & 3 & 0 \\
\hline 7745 & 3 & 3 & 3 & 3 & 0 & 9749 & 3 & 3 & 3 & 3 & 0 \\
\hline 7883 & 1 & 3 & 3 & 3 & 0 & 9830 & 3 & 3 & 3 & 3 & 0 \\
\hline 7994 & 3 & 3 & 3 & 3 & 0 & 9833 & 3 & 3 & 3 & 3 & 0 \\
\hline 8051 & 3 & 3 & 3 & 3 & 0 & 9869 & 3 & 3 & 3 & 3 & 0 \\
\hline 8057 & 3 & 3 & 3 & 3 & 0 & 9902 & 3 & 3 & 3 & 3 & 0 \\
\hline 8069 & 1 & 3 & 3 & 3 & 0 & 9905 & 3 & 3 & 3 & 3 & 0 \\
\hline 8255 & 3 & 3 & 3 & 3 & 0 & 9926 & 1 & $?$ & $?$ & 3 & 0 \\
\hline 8267 & 3 & 3 & 3 & 3 & 0 & 9995 & 1 & $?$ & 3 & 3 & 0 \\
\hline 8279 & 1 & 3 & 3 & 3 & 0 & & & & & & \\
\hline
\end{tabular}




\section{ACKNOWLEDGMENTS}

The authors express their gratitude to the referee who pointed out that the Theorem is not correct without the assumption that 3 does not split in $F / \mathbb{Q}$. We also express our gratitude to Mr. H. Sumida. We could correct some errors in Table 1 by comparing our data with his computational results.

\section{REFERENCES}

1. R. Greenberg, On the Iwasawa invariants of totally real number fields, Amer. J. Math. 98 (1976), 263-284. MR 53:5529

2. H. Hasse, Über die Klassenzahl abelscher Zahlkörper, Akademie Verlag, Berlin, 1952. MR 14:141

3. S. Mäki, The determination of units in real cyclic sextic fields, Lecture Notes in Math., vol. 797, Springer-Verlag, Berlin, Heidelberg, New York, 1980. MR 82a:12004

Department of Mathematics, College of Industrial Technology, Nihon University, 2-11-1 Shin-ei, NARAShino, Chiba, JaPAN

E-mail address: fukuda@math.cit.nihon-u.ac.jp

Department of Mathematics, Tokyo University of Agriculture and Technology, FUCHU, TOKYO, JAPAN 